\title{
An Unknown Input Moving Horizon Estimator for Open Channel Irrigation Systems
}

\author{
Gregory Conde, Nicanor Quijano, Senior Member, IEEE, and Carlos Ocampo-Martinez, Senior Member, IEEE
}

\begin{abstract}
The use of modeling and estimation strategies appears as a valuable tool to increase the efficiency of the open channel irrigation systems (OCIS). This paper is focused on exploring the feasibility, advantages, and conditions in the implementation of a moving horizon estimation (MHE) approach designed from an approximated model that contemplates mass and energy balances of the channels, which is useful to differentiate when a change of level is a conduction change effect, or when the change is due to an unknown input. The estimation strategy is evaluated via simulation using a test case reported in the literature. The results show that, with the use of the proposed estimation strategy, it is possible to reach an optimal estimation of the total amount of unknown inputs.
\end{abstract}

\section{INTRODUCTION}

The irrigation process demands nearly $70 \%$ of water that humanity is consuming, and most of this water is transported throughout open channel irrigation systems (OCIS). In OCIS, problems associated with unknown actions (in control terms, unknown inputs), such as leaks, seepage, and even robbery, dramatically reduce the system efficiency [1]. However, in OCIS, the problem of unknown inputs estimation has not received enough attention, and the use of recently simplified modeling strategies, and promising unknown input estimation techniques based on the moving horizon estimation (MHE) have not been explored. For example: in [2], two unknown input observers are designed assuming the OCIS as time-varying delayed state and inputs systems, using integrator delay as a modeling strategy, and diagnosing faults from residuals of the unknown observers; in [3], an observer-based control is designed from a linear simplification of the SaintVenant Equations (SVE); in [4], a fault detection strategy using linear parameter varying interval models is proposed; and in [5], two methods developed from a simplified volume balance model, and one method based on the SVE are presented. It is important to realize that in the reported works, most of the estimation strategies (i.e., [2]-[4]) have been

This research has been supported by Séptima Convocatoria Interna de Investigación de la Universidad Central, Convocatoria Proyectos de Investigación Conjunta Universidad de Ibagué-Universidad de los Andes, the CSIC Project MuYSCA (Ref. COOPA20246), and the Project DEOCS DPI2016-76493-C3-3R.

Gregory Conde is with Universidad de los Andes, Universidad Central, Bogotá, Colombia, and Universitat Politècnica de Catalunya, Barcelona, Spain (e-mail: gj.conde30@uniandes.edu.co).

Nicanor Quijano is with School of Engineering, Universidad de los Andes, Bogotá, Colombia (e-mail: nquijano@uniandes.edu.co).

Gregory Conde and Carlos Ocampo-Martinez are with Automatic Control Department, Universitat Politècnica de Catalunya, Institut de Robòtica i Informàtica Industrial (CSIC-UPC), Barcelona, Spain (e-mail: carlos.ocampo@upc.edu). developed from simplified or approximated models that do not contemplate energy balances along the channels, and this could lead to inaccurate estimations of the unknown inputs. This problem has been explained in [6], where, from test estimation strategies in a real system, drift in the results is reported, concluding that this drift is due to the growth of weeds, which affects the flow conduction. On the other hand, the direct use of the SVE contemplates the energy balances along the channels. However, as it is highlighted in [5], the direct use of the SVE for unknown input estimation design increases the design and implementation complexity of the estimation strategies.

Alternatively, showing high accuracy describing the OCIS behavior, in [7] an approximated modeling approach formulated from mass and energy balances has been proposed. This model appears as a potential solution in order to discriminate between levels variations due to unknown inputs and levels variations due to changes in flow conduction. Equally important, the moving horizon estimation (MHE) is a strategy that in recent years has received high attention. This estimation strategy can be formulated from a comprehensive description of the system, where the unknown parameters can be associated with uncertainties instead of residuals [8]. However, to the best of the authors' knowledge, the MHE strategy has not been reported for the estimation of unknown inputs in OCIS.

Given these points, this work is focused on the development and analysis of a strategy for OCIS that takes into account an MHE with unknown inputs, which is designed from the simplified model presented in [7]. As a result, the main contributions of this paper are twofold: i) the development of a new optimal unknown input estimation strategy for OCIS, which incorporates knowledge about mass and energy balances; and ii) the proposition of the necessary conditions to obtain optimal estimations of the unknown inputs. The performance of the unknown input estimation strategy is evaluated by simulation using the test case proposed in [9], and the results are contrasted with a Luenberger-based unknown input strategy also designed by using the model proposed in [7].

The remainder of the paper is organized as follows. Section II starts with a summary of the modeling strategy, and some proposed assumptions towards obtaining an estimation model are drawn. In Section III, the use of the MHE as an unknown input estimation strategy is explained. In Section IV, the necessary conditions to obtain optimal estimations 
of the unknown inputs are presented. In Section $\mathrm{V}$, the test case is explained, a comparison assessment is drawn, and the results are discussed. Finally, in Section VI, some conclusions are drawn.

\section{PROBLEM STATEMENT}

In the following, an overview of the model proposed in [7] is presented, which describes the behavior of the OCIS assuming two storage units per channel and performing mass and energy balances for each channel (see Figure 1).

\section{A. Modeling Approach}

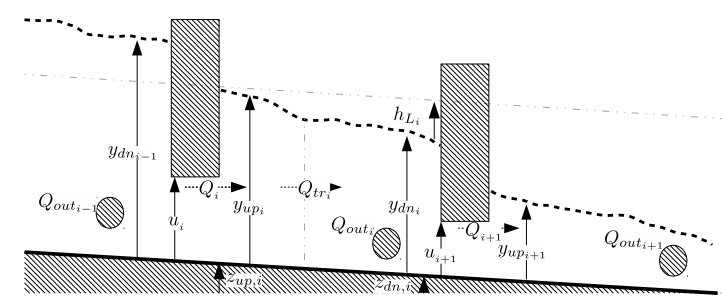

Fig. 1. Graphical description for the proposed energy and mass balances.

In this modeling approach, each channel is described with two differential equations of the form:

$$
\begin{aligned}
& A_{u p_{i}} \dot{y}_{u p_{i}}(t)=Q_{i}(t)-Q_{t r_{i}}(t) \\
& A_{d n_{i}} \dot{y}_{d n_{i}}(t)=Q_{t r_{i}}(t)-Q_{\text {out }_{i}}(t)-Q_{i+1}(t),
\end{aligned}
$$

where, for the $i^{\text {th }}$ channel, $y_{u p_{i}}(t)$ is the level at the upstream end of the channel, $y_{d n_{i}}(t)$ is the level at the downstream end of the channel, $A_{u p_{i}}$ and $A_{d n_{i}}$ are the areas of the assumed upper and lower storage units, $Q_{i}(t)$ is an inflow that comes from the upstream channel, $Q_{i+1}(t)$ is an outflow that feeds the downstream channel, $Q_{\text {out }_{i}}(t)$ are the outflows that feed either users or other channels. The static and dynamic behaviors of these flows are related to the kind of hydraulic structure that is used for flow regulation [10]. On the other hand, $Q_{t r_{i}}(t)$ is a flow transition between the assumed storage units. This flow can be obtained from an energy balance given by:

$$
z_{u p_{i}}+y_{u p_{i}}(t)+\frac{v_{u p_{i}}{ }^{2}}{2 g}=z_{d n_{i}}+y_{d n_{i}}(t)+\frac{v_{d n_{i}}{ }^{2}}{2 g}+h_{L_{i}}(t),
$$

where $v_{u p_{i}}$ and $v_{d n_{i}}$ are the upstream and downstream mean flow velocity, and $z_{u p_{i}}$ and $z_{d n_{i}}$ are the upstream and downstream elevations, respectively. $h_{L_{i}}(t)$ is known as the head lost due to friction described by $h_{L_{i}}(t)=f_{i}(t) \frac{L_{i} v_{i}{ }^{2}(t)}{D_{i} 2 g}$, where $L_{i}$ is the channel length, $D_{i}$ is the hydraulic diameter, and $f_{i}(t)$ is the friction factor. In this model, a constant friction factor and an equal mean flow velocity along the channel are assumed, obtaining that $h_{L_{i}}(t) \approx \frac{Q_{t r_{i}}^{2}(t)}{K_{t r_{i}}{ }^{2} y_{u p_{i}}^{2}(t)}$, where $K_{t r_{i}}$ is a transition constant coming from the analysis of the system in steady-state. Therefore, the flow transition is given by:

$$
\begin{aligned}
Q_{t r_{i}}(t) & =K_{t r_{i}} y_{u p_{i}}(t) \gamma_{i}(t) \\
\gamma_{i}(t) & =\sqrt{y_{u p_{i}}(t)-y_{d n_{i}}(t)+z_{u p_{i}}-z_{d n_{i}}} .
\end{aligned}
$$

\section{B. Model Assumptions}

In order to use the proposed model for the estimation of unknown inputs, some considerations should be made.

Assumption 1 (Transition constant): The friction factor is a function of the Reynolds number, which depends on the velocities of the fluid. In this work it is assumed that $K_{t r_{i}}=$ $\bar{K}_{t r_{i}}+\Delta K_{t r_{i}}(t)$, where $\bar{K}_{t r_{i}}$ is the value of the constant analysing the system in steady-state, and $\Delta K_{t r_{i}}(t)$ is the unknown variation of the transition constant.

Assumption 2 (Energy balance constant): In the steadystate and uniform flow cases, $\gamma_{i}(t)$ in Eq (3) can be considered as a constant [11]. Therefore, a linear description of the channel is assumed and $\gamma_{i}(t)=\bar{\gamma}_{i}+\Delta \gamma_{i}(t)$, where $\bar{\gamma}_{i}=\sqrt{\bar{y}_{u p_{i}}-\bar{y}_{d n_{i}}+z_{u p_{i}}-z_{d n_{i}}}$ can be obtained from measurements of the real system in steady-state, and $\Delta \gamma_{i}(t)$ is an unknown parameter.

Assumption 3 (Unknown inputs): The OCIS can be affected by unknown flows, such as seepage, leaks, and robbery. All of these inputs are assumed to be joint into the upstream unknown external inputs $\left(s_{u p_{i}}(t)\right)$, and the downstream unknown external inputs $\left(s_{d n_{i}}(t)\right)$.

Based on these three assumptions, the flow transition is given by:

$$
Q_{t r_{i}}(t)=\left(\bar{K}_{t r_{i}}+\Delta K_{t r_{i}}(t)\right) y_{u p_{i}}(t)\left(\bar{\gamma}_{i}+\Delta \gamma_{i}(t)\right),
$$

and the OCIS in (1) can be rewritten as:

$$
\begin{aligned}
A_{u p_{i}} \dot{y}_{u p_{i}}(t)= & Q_{i}(t)-\bar{K}_{t r_{i}} \bar{\gamma}_{i} y_{u p_{i}}(t)+\psi_{u p_{i}}(t) \\
A_{d n_{i}} \dot{y}_{d n_{i}}(t)= & \bar{K}_{t r_{i}} \bar{\gamma}_{i} y_{u p_{i}}(t)-Q_{o u t_{i}}(t)-Q_{i+1}(t)+ \\
& \psi_{d n_{i}}(t),
\end{aligned}
$$

where $\psi_{u p_{i}}(t)$ and $\psi_{d n_{i}}(t)$ are unknown parameters described by

$$
\begin{aligned}
& \psi_{u p_{i}}(t)=-\vartheta y_{u p_{i}}(t)+s_{u p_{i}}(t), \\
& \psi_{d n_{i}}(t)=\vartheta y_{u p_{i}}(t)+s_{d n_{i}}(t),
\end{aligned}
$$

where $\vartheta(t)$ is composed of the unknown parameters of the flow transition, i.e., $\vartheta(t)=\bar{K}_{t r_{i}} \Delta \gamma_{i}(t)+\Delta K_{t r_{i}}(t) \bar{\gamma}_{i}+$ $\Delta K_{t r_{i}}(t) \Delta \gamma_{i}(t)$.

\section{Estimation Formulation}

Considering that the dynamic behavior of the OCIS can be described using (5), an estimation model is proposed as

$$
\begin{aligned}
& A_{u p_{i}} \hat{\dot{y}}_{u p_{i}}(t)= Q_{i}(t)-\bar{K}_{t r_{i}} \bar{\gamma}_{i} \hat{y}_{u p_{i}}(t)+\hat{\psi}_{u p_{i}}(t) \\
& A_{d n_{i}} \hat{\dot{y}}_{d n_{i}}(t)= \bar{K}_{t r_{i}} \bar{\gamma}_{i} \hat{y}_{u p_{i}}(t) \\
&-Q_{o u t_{i}}(t)-Q_{i+1}(t)+\hat{\psi}_{d n_{i}}(t) \\
& \hat{\psi}_{u p_{i}}(t)=-\hat{\vartheta} y_{u p_{i}}(t)+\hat{s}_{u p_{i}}(t) \\
& \hat{\psi}_{d n_{i}}(t)=\hat{\vartheta} y_{u p_{i}}(t)+\hat{s}_{d n_{i}}(t),
\end{aligned}
$$

where $\hat{y}_{u p_{i}}(t)$, and $\hat{y}_{d n_{i}}(t)$ are the estimated upstream and downstream levels, and $\hat{\vartheta}, \hat{s}_{u p_{i}}(t)$, and $\hat{s}_{d n_{i}}(t)$ are the estimated unknown parameters. Subtracting (5) from (7), the estimation error is obtained as

$$
\begin{aligned}
& A_{u p_{i}} \dot{\hat{e}}_{u p_{i}}(t)=-\bar{K}_{t r_{i}} \bar{\gamma}_{i} \hat{e}_{u p_{i}}(t)+\psi_{u p_{i}}(t)-\hat{\psi}_{u p_{i}}(t) \\
& A_{d n_{i}} \dot{\hat{\hat{e}}}_{d n_{i}}(t)=\bar{K}_{t r_{i}} \bar{\gamma}_{i} \hat{e}_{u p_{i}}(t)+\psi_{d n_{i}}(t)-\hat{\psi}_{d n_{i}}(t),
\end{aligned}
$$


where $\hat{e}_{u p_{i}}(t)=y_{u p_{i}}(t)-\hat{y}_{u p_{i}}(t)$, and $\hat{e}_{d n_{i}}(t)=y_{d n_{i}}(t)-$ $\hat{y}_{d n_{i}}(t)$ are the upstream and downstream estimation errors, respectively. Following the discretization procedure for linear systems shown in [12], for a sampling time $\tau_{s}$, discrete descriptions of the estimation model and estimation error are given by

$$
\begin{aligned}
{\left[\begin{array}{c}
\hat{y}_{u p_{i}}(k+1) \\
\hat{y}_{d n_{i}}(k+1)
\end{array}\right] } & =G_{i}\left[\begin{array}{c}
\hat{y}_{u p_{i}}(k) \\
\hat{y}_{d n_{i}}(k)
\end{array}\right]+H_{i}\left[\begin{array}{c}
\hat{\psi}_{u p_{i}}(k) \\
\hat{\psi}_{d n_{i}}(k)
\end{array}\right] \\
& +H_{f_{i}}\left[\begin{array}{c}
Q_{i}(k) \\
Q_{\text {outi }}(k) \\
Q_{i+1}(k)
\end{array}\right]
\end{aligned}
$$

and

$$
\begin{aligned}
{\left[\begin{array}{c}
\hat{e}_{u p_{i}}(k+1) \\
\hat{e}_{d n_{i}}(k+1)
\end{array}\right] } & =G_{i}\left[\begin{array}{c}
\hat{e}_{u p_{i}}(k) \\
\hat{e}_{d n_{i}}(k)
\end{array}\right]-H_{i}\left[\begin{array}{c}
\hat{\psi}_{u p_{i}}(k) \\
\hat{\psi}_{d n_{i}}(k)
\end{array}\right] \\
& +H_{i}\left[\begin{array}{c}
\psi_{u p_{i}}(k) \\
\psi_{d n_{i}}(k)
\end{array}\right]
\end{aligned}
$$

where

$$
\begin{aligned}
G_{i}= & {\left[\begin{array}{cr}
e^{-\frac{\bar{K}_{t r_{i}} \bar{\gamma}_{i}}{A_{u p_{i}}} \tau_{s}} & 0 \\
\frac{A_{u p_{i}}}{A_{d n_{i}}}\left(1-e^{-\frac{\bar{K}_{t r_{i}} \bar{\gamma}_{i}}{A u p_{i}} \tau_{s}}\right) & 1
\end{array}\right], } \\
H_{i}= & {\left[\begin{array}{ccc}
\frac{1}{\bar{K}_{t r_{i}} \bar{\gamma}_{i}}\left(1-e^{-\frac{\bar{K}_{t r_{i} \bar{\gamma}_{i}}}{A_{u p_{i}}} \tau_{s}}\right. & \\
\frac{\tau_{s}}{A_{d n_{i}}}-\frac{A_{u p_{i}}}{A_{d n_{i}} K_{t r_{i}} \bar{\gamma}_{i}}\left(1-e^{-\frac{\bar{K}_{t r_{i}} \bar{\gamma}_{i}}{A_{u p_{i}}} \tau_{s}}\right) & \frac{\tau_{s}}{A_{d n_{i}}}
\end{array}\right], } \\
H_{f_{i}}= & {\left[\begin{array}{lll}
H_{i}(1,1) & 0 & 0 \\
H_{i}(2,1) & -\frac{\tau_{s}}{A_{d n_{i}}} & -\frac{\tau_{s}}{A_{d n_{i}}}
\end{array}\right] . }
\end{aligned}
$$

Besides, $G_{i}$ is a full-rank matrix, and considering that $y_{u p_{i}}(k)$ and $y_{d n_{i}}(k)$ are measured variables, it can be deduced that the system described by (10) is observable. Therefore, the dynamic behavior of $y_{u p_{i}}(k+1)$ and $y_{d n_{i}}$ can be reached by appropriate sequences of $\hat{\psi}_{u p_{i}}(k)$, and $\hat{\psi}_{d n_{i}}(k)$.

\section{PROPOSED APPROACH}

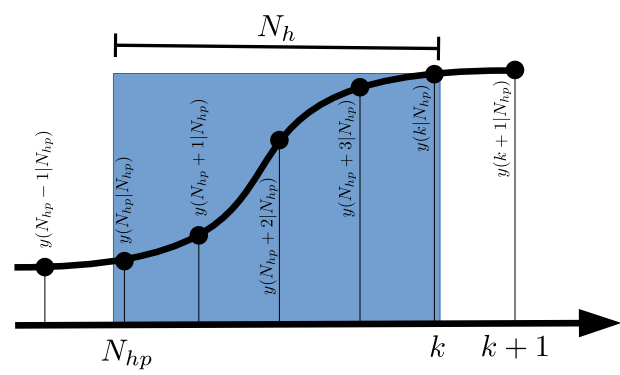

Fig. 2. Graphical description of data management along an estimation window.

In order to find suitable sequences of $\hat{\psi}_{u p_{i}}(k)$, and $\hat{\psi}_{d n_{i}}(k)$ that minimize the estimation errors $\hat{e}_{u p_{i}}(k+1)$, and $\hat{e}_{d n_{i}}(k+$ $1)$, an MHE strategy is considered. In this strategy, the estimation is formulated as a quadratic optimization problem over a window of data with fixed length [13]. Then, the objective is to find the unknown parameters of (8) (i.e., $\hat{\vartheta}$, $\hat{s}_{u p_{i}}, \hat{s}_{d n_{i}}$ ) that minimize the discrete estimation error (11). Therefore, as it is shown in Figure 2, for a sliding estimation window with length $N_{h}$ that starts in $N_{h p}=k-N_{h}+1$ and ends in $k$, along a moving horizon estimation, the solution of (10) is given by

$$
\begin{aligned}
\hat{\mathbf{Y}}_{\mathbf{i}}(\mathbf{k}+\mathbf{1})= & \boldsymbol{\Phi}_{\mathbf{i}}\left[\begin{array}{l}
\hat{y}_{u p_{i}}\left(N_{h p} \mid N_{h p}\right) \\
\hat{y}_{d n_{i}}\left(N_{h p} \mid N_{h p}\right)
\end{array}\right]+\mathbf{B}_{\mathbf{i}} \hat{\mathbf{\Psi}}_{\mathbf{i}}(\mathbf{k}) \\
& +\mathbf{B}_{\mathbf{f}_{\mathbf{i}}} \mathbf{Q}_{\mathbf{i}}(\mathbf{k}),
\end{aligned}
$$

where $\hat{\mathbf{\Psi}}_{\mathbf{i}}(\mathbf{k})=\boldsymbol{\Omega}_{\mathbf{i}}(\mathbf{k}) \hat{\boldsymbol{\Theta}}_{\mathbf{i}}(\mathbf{k})$, and $\hat{\mathbf{Y}}_{\mathbf{i}}(\mathbf{k}+\mathbf{1}), \boldsymbol{\Phi}_{\mathrm{i}}, \mathbf{B}_{\mathbf{i}}, \mathbf{B}_{\mathbf{f}_{\mathbf{i}}}$, $\mathbf{Q}_{\mathbf{i}}(\mathbf{k}), \hat{\boldsymbol{\Theta}}_{\mathbf{i}}(\mathbf{k})$, and $\boldsymbol{\Omega}_{\mathbf{i}}(\mathbf{k})$ are defined in the Appendix.

Then, in order to minimize the deviation of the estimated upstream and downstream levels from the measured levels, an MHE objective function is proposed as follows:

$$
\begin{aligned}
\mathbf{V}_{\mathbf{i}}(\mathbf{k}+\mathbf{1})= & \left\|\mathbf{Y}_{\mathbf{i}}(\mathbf{k}+\mathbf{1})-\hat{\mathbf{Y}}_{\mathbf{i}}(\mathbf{k}+\mathbf{1})\right\|_{\mathcal{R}_{1_{i}}}^{2}+ \\
& \left\|\boldsymbol{\Omega}_{\mathbf{i}}(\mathbf{k}-\mathbf{1}) \hat{\Theta}_{\mathbf{i}}(\mathbf{k}-\mathbf{1})-\boldsymbol{\Omega}_{\mathbf{i}}(\mathbf{k}) \hat{\Theta}_{\mathbf{i}}(\mathbf{k})\right\|^{2}{ }_{\mathcal{R}_{2_{i}}},
\end{aligned}
$$

where $\quad\left\|\mathbf{Y}_{\mathbf{i}}(\mathbf{k}+\mathbf{1})-\hat{\mathbf{Y}}_{\mathbf{i}}(\mathbf{k}+\mathbf{1})\right\|_{\mathcal{R}_{1_{i}}}^{2}$ accounts for the estimation error along an estimation window, $\left\|\boldsymbol{\Omega}_{\mathbf{i}}(\mathbf{k}-\mathbf{1}) \hat{\boldsymbol{\Theta}}_{\mathbf{i}}(\mathbf{k}-\mathbf{1})-\boldsymbol{\Omega}_{\mathbf{i}}(\mathbf{k}) \hat{\boldsymbol{\Theta}}_{\mathbf{i}}(\mathbf{k})\right\|^{2}{ }_{\mathcal{R}_{2}}$ is included as a forgetting factor that retains influence of past estimations [14], and $\hat{\boldsymbol{\Theta}}_{\mathbf{i}}(\mathbf{k}-\mathbf{1})$ is the sequence of parameters estimated in a previous iteration. Moreover, $\mathcal{R}_{1_{i}} \in \mathbb{R}^{2 N_{h} \times 2 N_{h}}$ and $\mathcal{R}_{2_{i}} \in \mathbb{R}^{2 N_{h} \times 2 N_{h}}$ are diagonal and positive definite weighting matrices that penalize the estimation error and the forgetting factor, respectively.

\section{MAIN THEORETICAL RESULTS}

Note that the reachability of suitable sequences of the unknown inputs $\left(\hat{\boldsymbol{\Theta}}_{\mathbf{i}}(\mathbf{k})\right)$ depends on the convexity of the objective function (13). In this section, in the next two lemmas and in the proposition, it is shown that an optimal estimation of $\hat{\boldsymbol{\Theta}}_{\mathbf{i}}(\mathbf{k})$ cannot be reached. However, the reachability of optimal sequences of the total amount of upstream and downstream unknown flows $\left(\hat{\mathbf{\Psi}}_{\mathbf{i}}(\mathbf{k})\right)$ can be guaranteed, and this property can be exploited in order to find the total amount of unknown flows $\left(s_{u p_{i}}+s_{d n_{i}}\right)$ that affect an open channel.

Lemma 1: From the objective function (13), only suboptimal estimations of $\hat{\boldsymbol{\Theta}}_{\mathbf{i}}(\mathbf{k})$ can be reached.

Proof: A necessary condition for any local minimum to be a global minimum is the convexity of the objective function (13). This condition can be reached if the Hessian with respect to $\hat{\Theta}_{\mathbf{i}}(k)$ of the objective function is positive definite, i.e.,

$$
\nabla^{2} \hat{\boldsymbol{\Theta}}_{\mathbf{i}}(k) \mathbf{V}_{\mathbf{i}}(k)=\boldsymbol{\Omega}_{\mathbf{i}}(k)^{\top} \mathbf{B}_{\mathbf{i}}^{\top} \mathcal{R}_{1_{i}} \mathbf{B}_{\mathbf{i}} \boldsymbol{\Omega}_{\mathbf{i}}(k)+\mathcal{R}_{2_{i}} \succ \mathbf{0} .
$$

Since $\mathcal{R}_{1_{i}}$ and $\mathcal{R}_{2_{i}}$ are positive defined, if $\boldsymbol{\Omega}_{\mathbf{i}}(k)^{\top} \mathbf{B}_{\mathbf{i}}^{\top} \mathbf{B}_{\mathbf{i}} \boldsymbol{\Omega}_{\mathbf{i}}(k) \succ \mathbf{0}$, the condition established in (14) is achieved.

A sufficient condition for $\boldsymbol{\Omega}_{\mathbf{i}}(k)^{\top} \mathbf{B}_{\mathbf{i}}^{\top} \mathbf{B}_{\mathbf{i}} \boldsymbol{\Omega}_{\mathbf{i}}(k) \succ \mathbf{0}$ is that the rank of $\mathbf{B}_{\mathbf{i}} \boldsymbol{\Omega}_{\mathbf{i}}(k)$ should be equal to $3 N_{h}$. But, 
given the dimensions of $\mathbf{B}_{\mathbf{i}}$ and $\boldsymbol{\Omega}_{\mathbf{i}}(k)$, the maximum rank of $\mathbf{B}_{\mathbf{i}} \boldsymbol{\Omega}_{\mathbf{i}}(k)$ is $2 N_{h}$. Therefore, the condition (14) and an optimal estimation of $\hat{\boldsymbol{\Theta}}_{\mathbf{i}}(k)$ cannot be reached.

However, by definition, $\boldsymbol{\Omega}_{\mathbf{i}}(k)^{\top} \mathbf{B}_{\mathbf{i}}^{\top} \mathbf{B}_{\mathbf{i}} \boldsymbol{\Omega}_{\mathbf{i}}(k)$ is positive semi-definite [15], then, the term

$$
\boldsymbol{\Omega}_{\mathbf{i}}(\mathbf{k})^{\top} \mathbf{B}_{\mathbf{i}}^{\top} \mathcal{R}_{1_{i}} \mathbf{B}_{\mathbf{i}} \boldsymbol{\Omega}_{\mathbf{i}}(\mathbf{k})
$$

is positive semi-definite.

Therefore, the Hessian $\left(\nabla^{2} \hat{\Theta}_{\mathbf{i}}(\mathbf{k}) \mathbf{V}_{\mathbf{i}}(k)\right)$ is positive semidefinite and only sub-optimal estimations of $\hat{\boldsymbol{\Theta}}_{\mathbf{i}}(\mathbf{k})$ can be guaranteed.

Lemma 2: From the objective function (13), an optimal estimation of $\hat{\mathbf{\Psi}}_{\mathbf{i}}(\mathbf{k})$ can be guaranteed.

Proof: Note that $\hat{\mathbf{\Psi}}_{\mathbf{i}}(\mathbf{k})$ is a linear combination of $\boldsymbol{\Omega}_{\mathbf{i}}(k)$ and $\hat{\boldsymbol{\Theta}}_{\mathbf{i}}(\mathbf{k})$. Then if the convexity of the objective function (13) is analyzed in function of $\hat{\mathbf{\Psi}}_{\mathbf{i}}(\mathbf{k})$, it can be established that (13) is convex if its Hessian with respect to $\hat{\Psi}_{\mathbf{i}}(\mathbf{k})$ is positive definite, i.e.,

$$
\nabla_{\hat{\mathbf{\Psi}}_{\mathbf{i}}(\mathbf{k})} \mathbf{V}_{\mathbf{i}}(\mathbf{k}+\mathbf{1})=\mathbf{B}_{\mathbf{i}}^{\top} \mathcal{R}_{1_{i}} \mathbf{B}_{\mathbf{i}}+\mathcal{R}_{2_{i}} \succ \mathbf{0} .
$$

In (15), $\mathcal{R}_{2_{i}}$ is positive definite, and if $\mathbf{B}_{\mathbf{i}}^{\top} \mathbf{B}_{\mathbf{i}} \succ \mathbf{0}$, the term $\mathbf{B}_{\mathbf{i}}^{\top} \mathcal{R}_{1_{i}} \mathbf{B}_{\mathbf{i}}$ is also positive definite. A sufficient condition for $\mathbf{B}_{\mathbf{i}}^{\top} \mathbf{B}_{\mathbf{i}} \succ \mathbf{0}$, is that the amount of linear combinations of $\mathbf{B}_{\mathbf{i}} \hat{\mathbf{\Psi}}_{\mathbf{i}}(\mathbf{k})$ should be equal to the length of $\hat{\mathbf{\Psi}}_{\mathbf{i}}(\mathbf{k})\left(2 N_{h}\right)$ or, in other words, that the rank of $\mathbf{B}_{\mathbf{i}}$ should be equal to $2 N_{h}$. Note that $G_{i}$ and $H_{i}$ are full-rank matrices, then it can be deduced that the rank of $\mathbf{B}_{\mathrm{i}}$ is equal to $2 N_{h}$. Therefore, an optimal estimation of $\hat{\mathbf{\Psi}}_{\mathbf{i}}(\mathbf{k})$ can be reached.

Lemma 1 shows that optimal estimations of the unknown parameters $\hat{\vartheta}, \hat{s}_{u p_{i}}$, and $\hat{s}_{d n_{i}}$ cannot be guaranteed. However, the unknown flow $\vartheta y_{u p_{i}}(k)$ associated with the uncertainty of the flow transition (6) presents a physical behavior that can be exploited: note that the unknown flow $\vartheta y_{u p_{i}}(k)$ leaves the upstream part of the channel and goes into the downstream part. Then, since optimal estimations of $\hat{\mathbf{\Psi}}_{\mathbf{i}}(\mathbf{k})$ can be guaranteed (Lemma 2), optimal estimations of the total amount of unknown flows $\left(s_{u p_{i}}+s_{d n_{i}}\right)$ that affect an open channel can be reached by

$$
\hat{\Psi}_{u p_{i}}(k)+\hat{\Psi}_{d n_{i}}(k)=s_{u p_{i}}(k)+s_{d n_{i}}(k) .
$$

Finally, in Proposition 1 the convergence rate of $\hat{\mathbf{\Psi}}_{\mathbf{i}}(\mathbf{k})$ can be established.

Proposition 1: If in the objective function (13), the forgetting factor is neglected $\left(\mathcal{R}_{2_{i}}=\mathbf{0}\right)$, an optimal estimation of $\hat{\Psi}_{\mathbf{i}}(\mathbf{k})$ will converge to $\Psi_{\mathbf{i}}(\mathbf{k})$ in one iteration.

Proof: In order that (10) and (11) have the same state matrix $G_{i}$, and the same input matrix $H_{i}$, from (12) it can be established that a receding estimation error is given by

$$
\begin{aligned}
\hat{\mathbf{e}}_{\mathbf{i}}(\mathbf{k}+\mathbf{1})= & \boldsymbol{\Phi}_{\mathbf{i}}\left[\begin{array}{c}
\hat{e}_{u p_{i}}\left(N_{h p} \mid N_{h p}\right) \\
\hat{e}_{d n_{i}}\left(N_{h p} \mid N_{h p}\right)
\end{array}\right]-\mathbf{B}_{\mathbf{i}} \hat{\Psi}_{\mathbf{i}}(\mathbf{k}) \\
& +\mathbf{B}_{\mathbf{i}} \boldsymbol{\Psi}_{\mathbf{i}}(\mathbf{k}),
\end{aligned}
$$

where

$$
\begin{aligned}
& \hat{\mathbf{e}}_{\mathbf{i}}(\mathbf{k}+\mathbf{1})=\left[\hat{e}_{u p_{i}}\left(N_{h p+1} \mid N_{h p}\right) \quad \hat{e}_{d n_{i}}\left(N_{h p+1} \mid N_{h p}\right) \cdots\right. \\
& \hat{e}_{u p_{i}}\left(N_{h p}+2 \mid N_{h p}\right) \quad \hat{e}_{d n_{i}}\left(N_{h p}+2 \mid N_{h p}\right) \cdots \\
& \left.\hat{e}_{u p_{i}}\left(k+1 \mid N_{h p}\right) \quad \hat{e}_{d n_{i}}\left(k+1 \mid N_{h p}\right)\right]^{\top} .
\end{aligned}
$$

Then, if the forgetting factor is neglected, an optimal solution of (13) is given by $\hat{\mathbf{e}}_{\mathbf{i}}(\mathbf{k}+\mathbf{1})=\mathbf{0}$. Therefore,

$$
\mathbf{B}_{\mathbf{i}} \hat{\Psi}_{\mathbf{i}}(\mathbf{k})=\boldsymbol{\Phi}_{\mathbf{i}}\left[\begin{array}{c}
\hat{e}_{u p_{i}}\left(N_{h p} \mid N_{h p}\right) \\
\hat{e}_{d n_{i}}\left(N_{h p} \mid N_{h p}\right)
\end{array}\right]+\mathbf{B}_{\mathbf{i}} \mathbf{\Psi}_{\mathbf{i}}(\mathbf{k}) .
$$

One step ahead, along an estimation window, an iterative solution of the estimation error is given by

$$
\begin{aligned}
\hat{\mathbf{e}}_{\mathbf{i}}(\mathbf{k}+\mathbf{1})= & \boldsymbol{\Phi}_{\mathbf{i}}\left[\begin{array}{c|c}
\hat{e}_{u p_{i}}\left(N_{h p-1} \mid N_{h p+1}\right) \\
\hat{e}_{d n_{i}}\left(N_{h p-1} \mid N_{h p+1}\right)
\end{array}\right]-\mathbf{B}_{\mathbf{i}} \hat{\mathbf{\Psi}}_{\mathbf{i}}(\mathbf{k}+\mathbf{1}) \\
& +\mathbf{B}_{\mathbf{i}} \boldsymbol{\Psi}_{\mathbf{i}}(\mathbf{k}+\mathbf{1}) .
\end{aligned}
$$

Note that in this step, $\left[\hat{e}_{u p_{i}}\left(N_{h p-1} \mid N_{h p+1}\right) \hat{e}_{d n_{i}}\left(N_{h p-1} \mid\right.\right.$ $\left.\left.N_{h p+1}\right)\right]^{\top}$ are the two first elements of $\hat{\mathbf{e}}_{\mathbf{i}}(\mathbf{k})$ with optimal solution $\hat{\mathbf{e}}_{\mathbf{i}}(\mathbf{k})=\mathbf{0}$ then $\boldsymbol{\Phi}_{\mathbf{i}}\left[\begin{array}{c}\hat{e}_{u p_{i}}\left(N_{h p-1} \mid N_{h p+1}\right) \\ \hat{e}_{d n_{i}}\left(N_{h p-1} \mid N_{h p+1}\right)\end{array}\right]=\mathbf{0}$.

Therefore, the optimal solution of (19) is given by $\hat{\mathbf{\Psi}}_{\mathrm{i}}(\mathbf{k}+\mathbf{1})=\boldsymbol{\Psi}_{\mathrm{i}}(\mathbf{k}+\mathbf{1})$.

The introduction of the forgetting factor allows preventing aggressive changes in the estimated parameters, which can be induced for disturbances such as measurement and process noise. Therefore, the use of a forgetting factor different from zero must be considered.

\section{TEST CASE}

The estimation strategies are validated using as a reference model, the test case introduced in [9], which has been proposed by the ASCE Task Committee on Canal Automation Algorithms as a standardized testbed case on canals with well-studied and realistic properties. Even though the test case is composed by 8 channels, since the estimation strategies present the same structure for any channel, the simulation is focused only on one channel (the first channel of the test case Figure 3). This is a rectangular channel with the following dimensions: length of $7000 \mathrm{~m}$, width of $6 \mathrm{~m}$, upstream elevation of $4.4 \mathrm{~m}$, and downstream elevation of $3.29 \mathrm{~m}$. In order to obtain data useful to test the estimation algorithm, the test case has been implemented in EPA-SWMM, which numerically solves the SVE of the hydraulic system, and the data of the simulation have been used as entries to the estimation algorithm, guaranteeing that the design and simulation models are different. Additionally, a measurement noise, normally distributed with mean 0 and variance 0.005 has been included in order to emulate the uncertainty that conventional level sensors present (e.g., ultrasound sensors). In the test case, the apertures of the inflow and outflow gates $\left(u_{1}, u_{2}\right)$ are kept fixed in the operation condition, with areas of $2.45 \mathrm{~m}^{2}$, and $8.24 \mathrm{~m}^{2}$, respectively. The source level is fixed in $5 \mathrm{~m}$, and the upstream and downstream levels of the eight channels are initialized at the steadystate. The generation of the unknown inputs is performed by variations of the orifices areas that limit the seepages. The parameters of the estimation model are obtained using Equations (3) and (5) without unknown disturbances and in steady-state where $Q_{i}=Q_{t r_{i}}$. The values of the areas $A_{u p_{i}}$ and $A_{d n_{i}}$ are obtained by data fitting, using Equation (10) and experimental data from the reference model, obtaining $A_{u p_{1}}=21864 \mathrm{~m}^{2}$ and $A_{d n_{1}}=27163 \mathrm{~m}^{2}$. On the other hand, 


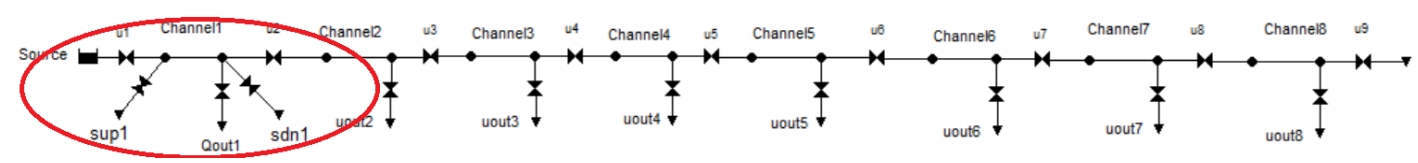

Fig. 3. Simulation diagram of the considered case study.

the sampling time has been obtained from the dominant dynamics of (5) following the recommendations presented in [10], i.e., $\tau_{s}=1000 \mathrm{~s}$.

\section{A. Simulation Results}

The MHE design process presents three elements to be tuned. The first one is the estimation window length $\left(N_{h}\right)$, the second one is the weighting matrix that penalizes the estimation error $\left(\mathcal{R}_{1_{i}}\right)$, and the third one is the weighting matrix that penalizes the forgetting factor $\left(\mathcal{R}_{2_{i}}\right)$. In order to find appropriate values for these parameters, a Monte Carlo experiment has been performed, where two key performance indicators (KPI) for the upstream, downstream, and unknown input estimation errors $\left(\hat{e}_{u p_{i}}(k), \hat{e}_{d n_{i}}(k)\right.$, and $\left.\hat{e}_{\psi_{i}}(k)\right)$ have been evaluated. The first KPI is the integral of the absolute error (IAE), which offers information about the mean behavior of the errors. The second KPI is the mean square error (MSE), which penalizes errors with large values. This kind of error occurs immediately after an unknown input change. Thus the MSE index accounts for overshoots and slow convergence rates. Concerning the estimation window length, the Monte Carlo experiment shows that variations of $N_{h}$ offer minor changes. On the other hand, variations between the weighting matrices ratios present remarkable results. In this direction, $\mathcal{R}_{1_{i}}$ has been established as a $2 N_{h} \times 2 N_{h}$ identity matrix, and the forgetting factor value has been established from the results of the Monte Carlo experiment, where the KPIs have been normalized and added obtaining the results shown in Figure 4. From this figure, an optimal forgetting factor of $0.027 I$ has been obtained.

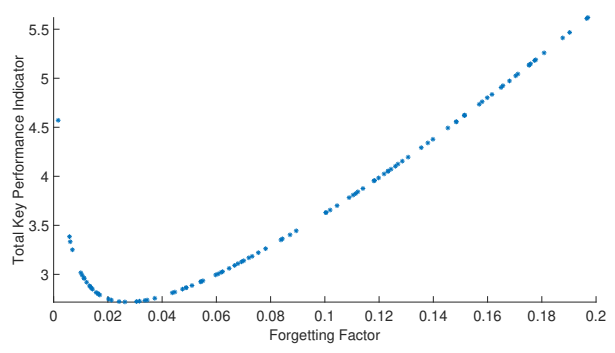

Fig. 4. Results of the Monte Carlo experiment for multiple forgetting factor values.

On the other hand, in order to contrast the obtained results, a Luenberger-based unknown input estimator has been designed. In this strategy, the objective is to estimate the unknown flows $\left(\hat{\Psi}_{i}\right)$ that guarantee an accurate estimation of the upstream and downstream levels. From (11), it can be deduced that if the steady-state estimation error converges to zero, the estimated unknown flows $\left(\hat{\Psi}_{i}\right)$ must converge to the unknown flows $\left(\Psi_{i}\right)$, and by using Equation (16) the total amount of unknown flows that affect an open channel can be reached. Therefore, in order to find the unknown flows
TABLE I

EVALUATION RESUlts OF THE MHE AND LUENBERGER ESTIMATORS

\begin{tabular}{|c|c|c|c|c|c|c|c|}
\hline \multirow[b]{2}{*}{$1 \times 10^{-3}$} & \multirow[b]{2}{*}{$\mathcal{R}_{2_{i}}$} & \multicolumn{5}{|c|}{ MHE } & \multirow[b]{2}{*}{ Luenberger } \\
\hline & & $\begin{array}{c}2 \times \\
10^{-1} I\end{array}$ & $\begin{array}{c}\times \\
10^{-1} I\end{array}$ & $\begin{array}{c}M T E \\
2.7 \times \\
10^{-2} I\end{array}$ & $\begin{array}{c}1 \times \\
10^{-3} I\end{array}$ & $\begin{array}{c}1 \times \\
10^{-4} I\end{array}$ & \\
\hline \multirow{2}{*}{$\hat{e}_{u p_{1}}$} & IAE & $9.9 \mathrm{~m}$ & $8.5 \mathrm{~m}$ & $8.4 \mathrm{~m}$ & $11.8 \mathrm{~m}$ & $12.6 \mathrm{~m}$ & $10.9 \mathrm{~m}$ \\
\hline & MSE & $0.04 \mathrm{~m}^{2}$ & $0.02 \mathrm{~m}^{2}$ & $0.01 \mathrm{~m}^{2}$ & $0.02 \mathrm{~m}^{2}$ & $0.02 \mathrm{~m}^{2}$ & $0.03 \mathrm{~m}^{2}$ \\
\hline \multirow{2}{*}{$\hat{e}_{d n_{1}}$} & IAE & $11.5 \mathrm{~m}$ & $9.8 \mathrm{~m}$ & $9.5 \mathrm{~m}$ & $12.5 \mathrm{~m}$ & $13.4 \mathrm{~m}$ & $22 \mathrm{~m}$ \\
\hline & MSE & $0.07 \mathrm{~m}^{2}$ & $0.03 \mathrm{~m}^{2}$ & $0.02 \mathrm{~m}^{2}$ & $0.02 \mathrm{~m}^{2}$ & $0.02 \mathrm{~m}^{2}$ & $0.2 \mathrm{~m}^{2}$ \\
\hline \multirow[t]{2}{*}{$\hat{e}_{\psi_{1}}$} & IAE & $227 \mathrm{~m}^{3} / \mathrm{s}$ & $130 \mathrm{~m}^{3} / \mathrm{s}$ & $95 \mathrm{~m}^{3} / \mathrm{s}$ & $389 \mathrm{~m}^{3} / \mathrm{s}$ & $555 \mathrm{~m}^{3} / \mathrm{s}$ & $245 \mathrm{~m}^{3} / \mathrm{s}$ \\
\hline & MSE & $19.7 \mathrm{~m}^{6} / \mathrm{s}^{2}$ & $10.5 \mathrm{~m}^{6} / \mathrm{s}^{2}$ & $4.6 \mathrm{~m}^{6} / \mathrm{s}^{2}$ & $23 \mathrm{~m}^{6} / \mathrm{s}^{2}$ & $47 \mathrm{~m}^{6} / \mathrm{s}^{2}$ & $10.8 \mathrm{~m}^{6} / \mathrm{s}^{2}$ \\
\hline
\end{tabular}

that ensure the convergence to zero of the estimation errors, in this estimator, the strategy guarantees the stability of the estimated integral of the upstream and downstream errors $\left(\hat{\xi}_{u p_{i}}(k)\right.$ and $\left.\hat{\xi}_{d n_{i}}(k)\right)$. In this order of ideas, the estimated unknown flows are given by $\hat{\Psi}_{i}=L_{i} \hat{E}_{i}(k)$, where $\hat{E}_{i}(k)=$ $\left[\hat{e}_{u p_{i}}(k) \hat{e}_{d n_{i}}(k) \hat{\xi}_{u p_{i}}(k) \hat{\xi}_{d n_{i}}(k)\right]^{\top}$ is the augmented vector of errors, and $L_{i} \in \mathbb{R}^{2 \times 4}$ has been found by pole placement [16], looking for the gain $L_{i}$ that ensures a dominant constant time estimation of $\left(10 \tau_{s}\right)$.

In Table I, five tests that evaluate the MHE forgetting factor incidence are contrasted with the Luenberger estimator. In this table, the upstream level estimation error $\left(\hat{e}_{u p_{1}}\right)$, the downstream level estimation error $\left(\hat{e}_{d n_{1}}\right)$, and the unknown input estimation error $\left(\hat{e}_{\Psi_{1}}\right)$ are considered.

From the evaluation test presented in Table I, it can be inferred that even in presence of noise and using realistic data, the proposed estimation strategies presents high performance. With the use of the proposed estimation strategies, it is possible to reach a mean upstream and downstream level estimation errors close to $9 \times 10^{-3} \mathrm{~m}$, and a mean unknown input estimation error close to $0.1 \mathrm{~m}^{3} / \mathrm{s}$. In this table, it is highlighted that the MHE presents better results using a forgetting factor of $2.7 \times 10^{-2} I$. This is due to the fact that large forgetting factor values reduce the variability of the estimated variables but increase the estimation convergence rates. On the other hand, lower forgetting factor values increase the convergence rate but increase the noise incidence increasing the variability of the estimated variables.

Note that the mean unknown input estimation error is higher than the mean upstream and downstream level estimation errors. This reflects one of the hardest problems of unknown inputs estimations in OCIS, where due to the usual large areas that these systems present, even large flow variations can be imperceptible from levels measurements, or can be masked between measurement and process noise.

Finally, the Luenberger estimator also exhibits good performance. This confirms the usefulness of the selected modeling strategy. However, in the Luemberger-based strategy, the mean of the unknown input estimation error is more than twice the mean of the estimation error of the MHE. This 
difference could be crucial if the estimated unknown inputs are used to calculate the system efficiency or for automatic control purposes.

\section{CONCLUDING REMARKS}

In this paper, an unknown inputs moving horizon estimation approach for open-channel irrigation systems has been presented. The estimation strategy has been designed from proposed considerations around a simplified non-linear model formulated from a mass and energy balance for each channel. Along the design process, the necessary conditions to obtain optimal estimations of the total amount of unknown inputs, and the convergence rate of the moving horizon estimation have been presented. The proposed estimation strategy has been tested using a benchmark of the literature, showing that, even though the estimator has been designed using a simplified modeling strategy, the unknown input estimation strategy is capable of accurately estimate the total amount of unknown inputs of an open channel. In the proposed strategy, the incorporation of a forgetting factor that retains the influence of past estimations has been considered and selected as a tuning parameter. The effects of the forgetting factor parameter have been evaluated using a Monte Carlo experiment and contrasted with a Luenbergerbased unknown input estimation strategy, showing that in most of the tests, the moving horizon strategy presents better performance than the Luenberger-based strategy. However, it must be highlighted that the Luenberger-based strategy could be an interesting option in situations where high performance is not demanded. Moreover, along the tests, the close relationship that the forgetting factor has with the noise incidence has been exposed. Moreover, it is important to realize that the development of new localization strategies, the incorporation of the proposed estimators with control strategies, and the real system implementation also are important future tasks.

\section{APPENDIX}

$$
\begin{aligned}
& \hat{\mathbf{Y}}_{\mathbf{i}}(\mathbf{k}+\mathbf{1})=\left[\hat{y}_{u p_{i}}\left(N_{h p}+1 \mid N_{h p}\right) \quad \hat{y}_{d n_{i}}\left(N_{h p}+1 \mid N_{h p}\right)\right. \\
& \hat{y}_{u p_{i}}\left(N_{h p}+2 \mid N_{h p}\right) \quad \hat{y}_{d n_{i}}\left(N_{h p}+2 \mid N_{h p}\right) \\
& \left.\cdots \hat{y}_{u p_{i}}\left(k+1 \mid N_{h p}\right) \quad \hat{y}_{d n_{i}}\left(k+1 \mid N_{h p}\right)\right]^{\top} \text {, }
\end{aligned}
$$

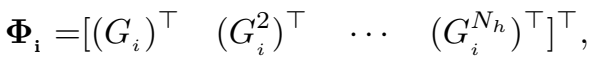

$$
\begin{aligned}
& \mathbf{B}_{\mathbf{i}}=\left[\begin{array}{ccc}
H_{i} & \mathbf{0} & \cdots \\
G_{i} H_{i} & H_{i} & \cdots \\
\vdots & \vdots & \vdots \\
G_{i}^{N_{h}-1} H_{i} & G_{i}^{N_{h}-2} H_{i} & \cdots
\end{array}\right] \\
& \mathbf{B}_{\mathbf{f}_{\mathbf{i}}}=\left[\begin{array}{ccc}
H_{i} & \mathbf{0} & \cdots \\
G_{i} H_{f_{i}} & H_{f_{i}} & \cdots \\
\vdots & \vdots & \vdots \\
G_{i}^{N_{h}-1} H_{f_{i}} & G_{i}^{N_{h}-2} H_{f_{i}} & \cdots
\end{array}\right], \\
& \mathbf{Q}_{\mathbf{i}}(\mathbf{k})=\left[Q_{i}\left(N_{h p} \mid N_{h p}\right) \quad Q_{\text {out }_{i}}\left(N_{h p} \mid N_{h p}\right) \quad \ldots\right. \\
& Q_{i}\left(N_{h p}+1 \mid N_{h p}\right) \quad Q_{\text {out }_{i}}\left(N_{h p}+1 \mid N_{h p}\right) \quad \ldots \\
& \left.Q_{i}\left(k \mid N_{h p}\right) \quad Q_{\text {out }_{i}}\left(k \mid N_{h p}\right) \quad Q_{i+1}\left(k \mid N_{h p}\right)\right]^{\top} \text {, }
\end{aligned}
$$

$$
\begin{aligned}
& \hat{\boldsymbol{\Theta}}_{\mathbf{i}}(\mathbf{k})=\left[\hat{\vartheta}\left(N_{h p} \mid N_{h p}\right) \quad \hat{s}_{u p_{i}}\left(N_{h p} \mid N_{h p}\right) \hat{s}_{d n_{i}}\left(N_{h p} \mid N_{h p}\right)\right. \\
& \hat{\vartheta}\left(N_{h p}+1 \mid N_{h p}\right) \quad \hat{s}_{u p_{i}}\left(N_{h p}+1 \mid N_{h p}\right) \quad \ldots \\
& \left.\hat{\vartheta}\left(k \mid N_{h p}\right) \quad \hat{s}_{u p_{i}}\left(k \mid N_{h p}\right) \hat{s}_{d n_{i}}\left(k \mid N_{h p}\right)\right]^{\top} \\
& \boldsymbol{\Omega}_{\mathbf{i}}(\mathbf{k})=\left[\begin{array}{ccc}
\alpha_{i}\left(N_{h p} \mid N_{h p}\right) & \mathbf{0} & \mathbf{0} \\
\mathbf{0} & \ddots & \mathbf{0} \\
\mathbf{0} & \mathbf{0} & \alpha_{i}\left(k \mid N_{h p}\right)
\end{array}\right] \text {, }
\end{aligned}
$$

where $\mathbf{0}$ is a matrix of suitable dimensions with null entries, and

$$
\alpha_{i}\left(N_{h p} \mid N_{h p}\right)=\left[\begin{array}{ccc}
-y_{u p_{i}}\left(N_{h p} \mid N_{h p}\right) & 1 & 0 \\
y_{d n_{i}}\left(N_{h p} \mid N_{h p}\right) & 0 & 1
\end{array}\right] .
$$

\section{REFERENCES}

[1] A. Moavenshahidi, R. Smith, and M. Gillies, "Seepage losses in the Coleambally Irrigation Area - loss estimates from channel automation data," Australian Journal of Water Resources, vol. 20, no. 1, pp. 78-88, 2016.

[2] D. Koenig, N. Bedjaoui, and X. Litrico, "Unknown Input Observers Design for Time-Delay Systems Application to An Open-Channel," in Proceedings of the 44th IEEE Conference on Decision and Control, 2005 , pp. 5794-5799.

[3] G. Besançon, J.-F. Dulhoste, and D. Georges, "Nonlinear observerbased feedback for open-channel level control," Journal of Hydraulic Engineering, vol. 134, no. 9, pp. 1267-1274, 2008.

[4] J. Blesa, V. Puig, and Y. Bolea, "Fault detection using interval LPV models in an open-flow canal," Control Engineering Practice, vol. 18, no. 5, pp. 460-470, 2010.

[5] N. Bedjaoui and E. Weyer, "Algorithms for leak detection, estimation, isolation and localization in open water channels," Control Engineering Practice, vol. 19, no. 6, pp. 564-573, 2011.

[6] N. Bedjaoui, X. Litrico, D. Koenig, J. Ribot-Bruno, and P.-O. Malaterre, "Static and dynamic data reconciliation for an irrigation canal," Journal of Irrigation and Drainage Engineering, vol. 134, no. 6, pp. 778-787, 2008.

[7] G. Conde, N. Quijano, and C. Ocampo-Matinez, "Control-Oriented Modeling Approach for Open Channel Irrigation Systems," in Proceedings of the IFAC 21s World Congress, Berlin, Germany, 2020.

[8] G. Franze and D. Famularo, "Robust Fault Detection for Constrained Uncertain Linear Systems: A Moving Horizon Estimation Approach," in Proceedings of the IEEE Conference on Decision and Control, 2018, pp. $5574-5579$.

[9] A. Clemmens, T. Kacerek, B. Grawitz, and W. Schuurmans, "Test Cases for Canal Control Algorithms," Journal of Irrigation and Drainage Engineering, vol. 124, pp. 23-30, 1998.

[10] X. Litrico and V. Fromion, Modeling and Control of Hydrosystems. London: Springer, 2009.

[11] M. Chaudhry, Open-Channel Flow, 2nd ed. Columbia: Springer Science+Business Media, 2008.

[12] C. T. Chen, Linear System Theory and Design, ser. Oxford series in electrical and computer engineering. Oxford University Press, 2013.

[13] P. Segovia, L. Rajaoarisoa, F. Nejjari, E. Duviella, and V. Puig, "Model predictive control and moving horizon estimation for water level regulation in inland waterways," Journal of Process Control, vol. 76, pp. 1-14, 2019.

[14] J. Baillieul and T. Samad, Encyclopedia of Systems and Control, ser Encyclopedia of Systems and Control, J. Baillieul and T. Samad, Eds. London: Springer International Publishing, 2015.

[15] R. Bhatia, Positive Definite Matrices. Princeton University Press, 2015.

[16] F. Bakhshande and D. Söffker, "Proportional-Integral-Observer: A brief survey with special attention to the actual methods using ACC Benchmark," in Proceedings of the 8th Vienna International Conferenceon Mathematical Modelling MATHMOD 2015, vol. 28, no. 1. Elsevier Ltd., 2015, pp. 532-537. 